\title{
Time-resolved pulsed stimulated infrared thermography applied to carbon/carbon non destructive evaluation
}

\author{
by Ph.M. DELPECH, D.M. BOSCHER, F. LEPOUTRE \\ A.A. DEOM and D.L. BALAGEAS
}

ONERA, Thermophysics Division, BP 72, F-92322 CHATILLON-Cedex, France.

\begin{abstract}
The pulsed stimulated infrared thermography is used for the non destructive evaluation of the thermal protection of the European space shuttle Hermès. The hottest parts of the thermal protection are made of coated carbon/carbon composite materials which is a rather good thermal conductor. After a brief presentation of the model and of the identification procedure, results are compared with micrography examinations and Compton back scattering showing a good agreement between the different methods.
\end{abstract}

\section{Nomenclature}

index $i$ refer to layer $i$

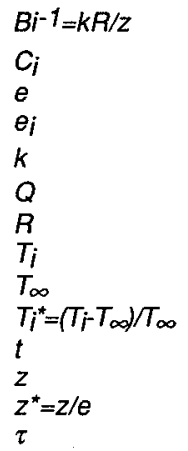

inverse of Biot number
volumic heat capacity
total thickness of the sample
thickness (index i refers to layer i)
thermal conductivity
fluence
thermal resistance
temperature
adiabatic temperature
adimensional temperature
time
depth
adimensional depth
pulse duration

$J . m^{-3} \cdot K^{-1}$
$m$
$m$
$W \cdot m^{-1} \cdot K^{-1}$
$J . m^{-2}$
$m^{2} \cdot K \cdot W^{-1}$
$K$
$K$
$s$
$m$
$s$

\section{Introduction}

Among all the non destructive evaluation (NDE) techniques, Stimulated Infrared Thermography (SIRT) is very attractive because of its non contact and rapid-scanning ability [1]. We applied this technique to composite materials which are often used in aerospace structures, in particular to carbon/epoxy composites [2,3]. Due to the relatively low refreshment frequency of current IR cameras, the application of the method was restricted to low thermal conductors. Recent developments of the data reduction procedure [4] allow to apply SIRT to good heat conductors. Satisfactory results were obtained in the case of delaminations in carbon/carbon $(\mathrm{C} / \mathrm{C})$ composites which will constitute the hottest parts of the thermal protection of the European space shuttle Hermès. More recently, an improvement of the method was presented [5] with application to adhesive bonds in metallic structures. Here we present results related to Hermès $\mathrm{C} / \mathrm{C}$ composites using the new reduction procedure.

After a brief presentation of the experimental set up, the model and the identification procedure are explained. Results are given and compared with electron micrography examination, used as a destructive reference method, and with Compton back scattering. Finally, a comparison is made between the present data reduction procedure and the one developed for low conductivity 


\section{http://dx.doi.org/10.21611/qirt.1992.030}

materials as carbon/epoxy composites. The compared accuracies and possible fields of application are determined.

\section{SIRT experiments}

The sample used in this study is a $200 \mathrm{~mm}$ square plate of $\mathrm{C} / \mathrm{C}$ composite material elaborated for Hermès by Aérospatiale. This $3 \mathrm{~mm}$ thick sample contains natural defects in the core material. The defects to be detected and characterized are voids with a very small thickness compared to their extend.

For our SIRT experiments, we use a strong source of radiative energy constituted of 9 IR lamps (18kW total electrical power). The temperature distribution of the sample surface following the heat pulse (typical duration 0.6s) is monitored by an IR camera Agema 880 LWB. The signal is digitized in real time and recorded on the hard disc of the micro-computer which supervises the experiment.

\section{Data reduction procedure used for $\mathrm{c} / \mathrm{c}$ composites}

\subsection{The isothermal 1D model}

Let us consider a two layer sample of total thickness e, with a non perfect thermal contact at the interface (the defect), located at a depth $e_{1}$ (see figure ta). We set: $e_{2}=e_{-} e_{1}$, and $C_{1}, C_{2}$ the volumic heat capacities of the first and second layers respectively. At every time after the pulsed heat deposition, the temperature distribution can be represented by the sketch of figure 1b. If we take into account the fact that the thermal resistance of the defect is much larger than the thermal resistances of both layers (which have high conductivities), each layer may be considered as isothermal, with temperatures $T_{1}(t)$ in the first layer and $T_{2}(t)$ in the second one. With these assumptions, assuming no heat losses after the pulse, the front surface temperature of the sample, which is also the temperature of the first layer, is:

$$
T_{1 D}(t)=T_{\infty}[1+q \exp (-s t)]
$$

for a Dirac pulse. Here : $T_{\infty}=\frac{Q}{C_{1 e_{1}+C_{2} e_{2}}}, s=\frac{C_{1 e_{1}+C_{2} e_{2}}}{R C_{1} e_{1} C_{2} e_{2}}, q=\frac{C_{2 e_{2}}}{C_{1 e_{1}}}, Q$ being the fluence.

This solution can be extended to the case of a square pulse of finite duration $\tau$ (Duhamel theorem) and this temperature $T_{1}$ can be normalized $\left(T_{1}{ }^{*}\right)$ using the adiabatic temperature $T_{\infty}$ reached at very large times:

$$
T_{1}^{*}=\frac{T_{1}-T_{\infty}}{T_{\infty}}=\frac{q}{s \tau}[\exp (s \tau)-1] \exp (-s t)
$$

A data reduction procedure can be proposed for the identification of the depth $e_{1}$ and the thermal resistance $R$ of the defect. According to eq. (2), the thermogram, in semilogarithmic scale, $\log \left(T_{1}{ }^{*}\right)=\log \left(T_{1}^{*}(t=0)\right)-s t$, is a straight line of slope:

$$
-s=-\left(C_{1} e_{1}+C_{2} e_{2}\right) / R C_{1} e_{1} C_{2} e_{2}
$$

and of ordinate at the origin :

$$
T_{1}^{*}(0)=\frac{q}{s \tau}[\exp (s \tau)-1]
$$

The depth of the interface verifies the relation :

$$
e_{1}=\frac{e}{1+\frac{C_{1}}{C_{2}} \frac{\tau T_{1}(0) s}{\exp (s \tau)-1}},
$$

which allows an iterative determination of the depth $e_{1}$, once $s$ and $T_{1}^{*}(t=0)$ are experimentally determined in the graph $\log T_{1}^{*}=f(t)$. The procedure starts with the value $e_{1}=e / 2$ and stops when two successive values are close enough. Practically, five iterations are sufficient for a convergence better than $10^{-3}$. Finally, the thermal resistance $R$ characterizing the defect is given by the relation: 


$$
R=\frac{C_{1} e_{1}+C_{2} e_{2}}{S C_{1} e_{1} C_{2} e_{2}}
$$

\subsection{Procedure to estimate the asymptotic adiabatic temperature $\mathrm{T}_{\infty}$}

The method needs to evaluate the asymptotic temperature $T_{\infty}$. Practically $T_{\infty}$ cannot be determined easily from the thermogram because, due to the high value of the thermal resistance, the time to reach it is important, allowing the free convection losses to introduce noticeable perturbations.

Actually, it is always possible to determine $T_{\infty}$ from the temperature $T_{1}(t)$ at any time $t$ provided that, (i) heat losses have not affected $T_{1}(t)$ and, (ii) the signal to noise ratio is large enough. To improve the first point (i), we stop the analysis of the thermogram at a time tmax. It is difficult to find a universal criterion for the choice of tmax. Generally, we study the thermogram of a particular pixel and we choose tmax as the limit beyond which appears the temperature decrease which is characteristic of the beginning of the heat losses. To improve the second point (ii), let us recall that our experimental thermogram is given by :

$$
T_{1}(t)=T_{\infty}+b . e^{(-s . t)}
$$

from which we have to deduce $T_{\infty}$.

If we call $T_{1 \max }$ the temperature at $\mathrm{t}=\mathrm{t}_{\max }$, we can write :

$$
\left.\Delta T=T_{1}-T_{1} \max =b .\left(e^{(-s . t)}-e^{(-s . t m a x}\right)\right)
$$

which is a function of only $b$ and $s$.

Now we calculate a weighted integral of $\Delta T$ :

$$
I=\int_{t_{\text {min }}}^{t_{\max }} \Delta T e^{(-p . t)} d t
$$

in which $t_{\min }$ is roughly the time of the end of the heat pulse. The purpose of this integral is to reduce the noise by increasing the weight of the temperature at the beginning of the thermogram and, on the contrary, to decrease the weight of the end of the thermogram which is much more noisy.

Finally to eliminate $b$ from equation (3), it is necessary to evaluate I for two values of $p\left(p_{1}\right.$ and $\left.p_{2}\right)$ and to calculate the ratio $I\left(p_{1}\right) / I\left(p_{2}\right)$ which depends only on $s$. The choice of $p_{1}$ and $p_{2}$ is not critical, but these two values must be chosen as different as possible, keeping in mind that if $p_{2}$ is too large the calculation of $I\left(p_{2}\right)$ will not be an exact integral but, actually, a finite sum of a few images : we generally take $p_{1}=1 / t_{\max }$ and $p_{2}=2 p_{1}$.

Practically, the procedure begins with the identification of $s$ by comparing the experimental value of $I\left(p_{1}\right) / l\left(p_{2}\right)$ and the analytical value of this ratio. Then $b$ is deduced from $I\left(p_{1}\right)$ and finally $T_{\infty}$ is estimated from the average value of the temperature at a particular time. We have shown with numerical simulations that this procedure reproduces quite well the complete thermogram even if $T_{\infty}$ is experimentally totally unknown.

\section{Quantitative images of defects in coated carbon-carbon samples}

\subsection{Depth and thermal resistance images}

The data reduction procedure previously described is applied for each pixel of the successive thermal images obtained after the pulse. Figure 2 presents such a temperature image obtained on the Hermes material. For each pixel, the previously described data reduction is applied. From the successive thermal images it is then possible to calculate a depth image and a thermal resistance image which characterize completely the existing defects in the tested sample. Such synthetic images are given in color figures $A^{*}$ and $B$.

\subsection{Comparison with electron micrography examination}

In order to validate quantitatively these results, it was necessary to get reference informations from a micrography examination. The sample was cut in six different sites chosen on defects 


\section{http://dx.doi.org/10.21611/qirt.1992.030}

detected by thermography. The micrographies were performed using a scanning electron microscope with a magnification $\times 30$. The figure 3 shows a schematic representation of such a micrography. They allow a direct evaluation of the depth and the thickness of the defects. The depth profiles are in agreement with the hypothesis that delaminations and defects can be assimilated to thin layers of air in comparison with their spatial extend. The results are given in the following table for two defects.

\begin{tabular}{|c|c|c|c|}
\hline & & defect 1 & defect 2 \\
\hline \multirow{3}{*}{ Depth $(\mathrm{mm})$} & NDE & 1.2 & 0.88 \\
\cline { 2 - 4 } & micrography & 1.4 & 0.80 \\
\cline { 2 - 4 } & discrepancy & $14 \%$ & $10 \%$ \\
\hline Equivalent air & NDE & 90 & 39 \\
\cline { 2 - 4 } gap thickness $(\mu \mathrm{m})$ & micrography & 100 & 20 \\
\cline { 2 - 4 } & discrepancy & $10 \%$ & $95 \%$ \\
\hline
\end{tabular}

Table : compared accuracy between NDE by SIRT and destructive micrography examination

\subsection{Comparison with Compton back scattering}

A Compton back scattering experiment has been performed on the same sample. This method is currently used with composite materials as a characterization and non destructive testing method. It is able to achieve, with a good accuracy, local thickness and density measurements. The principle of the method is to count photons back scattered by a small volume inside the sample defined by the incident $X$ beam and the detector direction of sight [6]. Detection of defects is based on the $X$ attenuation differences between air and the material. This method seems to be very interesting because as the SIRT it is a one side method and it can be applied to any material. Associated to a scanning system, it is possible to realize two-dimensional and threedimensional images. Such an image, shown on figure 4, is obtained at a particular depth of $1 \mathrm{~mm}$ and is similar to theSIRT images (Figure 2). However, the image presented here is not so detailed than the SIRT image, especially the little defects which are closer from the front face than the big one, are not well identified. For a complete identification of defects it is necessary to perform an image for each particular depth where the information is needed. Such technique is more time consuming than SIRT.

\section{Comparison with the effusivity method and respective fields of application}

\subsection{The effusivity method}

Let us recall the principle of the effusivity method applied. This method, which applies to rather low conductors materials like carbon/epoxy composites, assumes that the sample can be assimilated to a semi infinite medium, without losses. For a very short pulse, the front face temperature is given by:

$$
\Delta \mathrm{T}(\mathrm{t})=\frac{\mathrm{Q}}{\mathrm{b}_{0} \sqrt{\pi \mathrm{t}}}
$$

where $Q$ is the absorbed fluence and $b_{O}$ is the effusivity of the material. When a defect is present (thermal resistance $R$ at depth $z$ ) the temperature does not follow the previous relation. One can define an apparent effusivity (depending on time) by:

$$
\mathrm{b}(\mathrm{t})=\frac{\mathrm{Q}}{\Delta \mathrm{T} \sqrt{\pi \mathrm{t}}}
$$

The minimum value of the ratio $b(t) / b_{0}$ vs time and the time at which it occurs are characteristics of the defect [3]. These values allow the depth $z$ and the thermal resistance $R$ to be calculated.

\subsection{Compared accuracies}

In order to determine the accuracy of both methods we defined the following adimensional parameters :

$$
. z^{*}=z / e, \text { depth of the defect divided by the total thickness of the sample }
$$


$B i^{-1}=k R / z$, reciprocal of the Biot number where $\mathrm{k}$ is the conductivity of the material and $R$ is the thermal resistance of the defect. Thermograms of the front surface illuminated by a Dirac pulse were calculated for different values of these parameters. From them, $\mathrm{Z}^{*}$ and $\mathrm{Bi}-1$ were identified using both described methods (section 3.1 and 5.1).

As shown in figures $5 . a$ and $5 . b$, both methods have clearly complementary fields of application. The first method based on the isothermal model leads to good results for the highest value of $B i-1$, i.e. the highest values of the defect thermal resistance. Indeed for those cases the isothermal hypothesis is verified at best. The identification is more accurate for $z^{*}$ than for $B i^{-1}$. This second result is due to the correlation between the two paramaters ( $B i^{-1}$ depending on $z$ ). On the contrary for the second method the accuracy is better for the lowest values of $\mathrm{Bi}^{-1}$ but is much more dependent on the value of $z^{*}$ (here $z^{*}=0.4$ ).

\section{Conclusion}

The possibilities of the pulsed stimulated thermography as a non-destructive testing method for good conductor materials was demonstrated. The application on carbon-carbon composites shows that SIRT can be considered as a suitable industrial NDE method. However, the quantitative evaluation involves to choose an adapted characterization method taking into account the thermophysical properties of the material and the expected characteristics of the defects. The 1D inversion procedure presented in this paper for good conductors leads to satisfactory results in a rather short time provided that the hypothesis about defects are verified. Otherwise, it may be considered as the first quantitative step of an iterative procedure which takes into account heat losses and finite dimensions of defects.

\section{Acknowledgement}

The financial support of this work under a Aérospatiale grant is gratefully acknownledged.

\section{REFERENCES}

[1] POTET (P.), BALAGEAS (D.L.), DEOM (A.A.) and BOSCHER (D.M.). - The PTR 8900: an industrial apparatus for testing materials by infrared photothermography. in Review of Progress in Quantitative NDE, edited by D.O. Thompson and D.E. Chimenti, Plenum Press, New York, 1990 Vol. 9A, p. 1017-1023.

[2] BALAGEAS (D.L.), DEOM (A.A.) and BOSCHER (D.M.). - Characterization and nondestructive testing of carbon-epoxy composites by a pulsed photothermal method in Materials Evaluations 45, 461, 1987.

[3] DEOM (A.A.), BOSCHER (D.M.) and BALAGEAS (D.L.). - Pulsed photothermal nondestructive testing - Application to carbon epoxy laminates in Review of Progress in Quantitative NDE, edited by D.O. Thompson and D.E. Chimenti, Plenum Press, New York, 1990, Vol. 9A, p. 525-531.

[4] BALAGEAS (D.L.), DELPECH (P.M.), BOSCHER (D.M.) and DEOM (A.A.). - New developments in stimulated infrared thermography applied to non destructive evaluation of

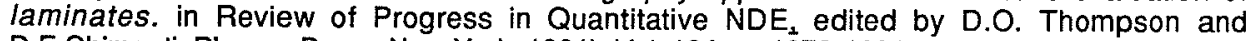
D.E.Chimenti, Plenum Press, New York, 1991), Vol. 10A, p. 1073-1081.

[5] DELPECH (P.M.), BOSCHER (D.M.), LEPOUTRE (F.), DEOM (A.A.) and BALAGEAS (D.L.). - Non destructive evaluation of adhesive bonds by pulse-stimulated infrared

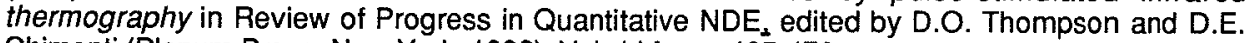
Chimenti (Plenum Press, New York, 1992), Vol. 11A, pp. 465-470.

[6] BABOT (D.), PEIX (G.), DUVAUCHELLE (P.), LE FLOC'H (C.) and SARRAZIN (P.). Thickness and density measurement of advanced materials by using backscattering of $X$-rays in Review of Progress in Quantitative NDE ${ }_{1}$ edited by D.O. Thompson and D.E.Chimenti, Plenum Press, New York, 1991, Vol. 10A, p. 385-392. 


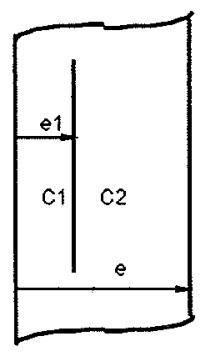

1.a

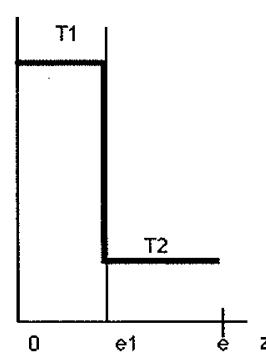

1.b

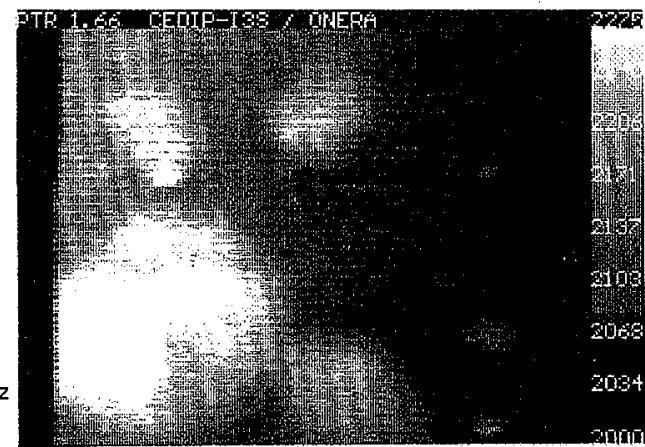

Fig.1. - Model used for defect identification in the case of high conductive materials. 1.a : Two-layers sample (subscripts 1 and 2) with non perfect contact at the interface; 1.b : Temperature profile in the sample of fig.1.a after a pulsed heat deposition at $z=0$.

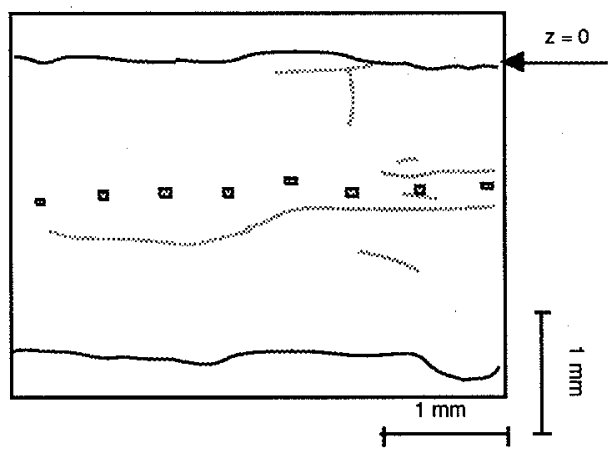

Fig. 3.- schematic representation of a micrography of a cross-section a-a. depth identified by thermography crack seen on the micrography

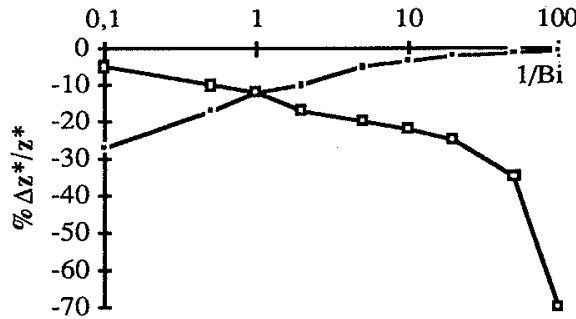

5.a.

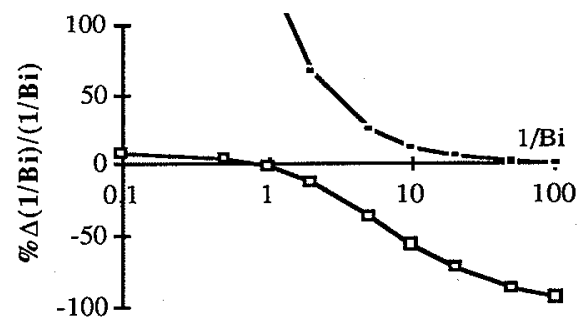

5.b.

Fig. 5. - Relative error in percent on the identification of $\mathrm{z}^{\star}$ (5.a.) and $\mathrm{Bi}^{-1}$ (5.b.) as a function of $\mathrm{Bi}^{-1}$ by the isothermal method (plain symbols) and the effusivity method (void symbols). The nominal value of $z^{\star}$ is 0.4 . 